\title{
FUZZY BAYESIAN INFERENCE
}

\author{
REINHARD VIERTL \\ Institut fuer Statistik \\ Technische Universitaet Wien \\ e-mail: R.Viertl@tuwien.ac.at
}

\begin{abstract}
Data are frequently not precise numbers but more or less non-precise, also called fuzzy. Moreover a-priori information in Bayesian inference is usually not available as a precise probability distribution. In case of fuzzy data and fuzzy a-priori information Bayes' theorem has to be generalized. There are different approaches for a generalization of Bayes' theorem but most of them don't keep the sequential updating of standard Bayesian inference. A generalization taking care of this is possible and will be explained in the talk. Also an alternative definition of fuzzy predictive distributions based on the so-called fuzzy probability integral will be given.
\end{abstract}

